\title{
Primary right atrial angiosarcoma mimicking acute pericarditis, pulmonary embolism, and tricuspid stenosis
}

\author{
K Ananthasubramaniam, A Farha
}

\begin{abstract}
A 29 year old white man presented to the emergency room with new onset pleuritic chest pain and shortness of breath. He was initially diagnosed as having viral pericarditis and was treated with non-steroidal anti-inflammatory drugs. A few weeks later he developed recurrent chest pain with cough and haemoptysis. Chest radiography, cardiac examination, transthoracic and transoesophageal echocardiography pointed to a mass that arose from the posterior wall of the right atrium, not attached to the interatrial septum, which protruded into the lumen of the right atrium causing intermittent obstruction of inflow across the tricuspid valve. Contrast computed tomography of the chest showed a right atrial mass extending to the anterior chest wall. The lung fields were studded with numerous pulmonary nodules suggestive of metastases. A fine needle aspiration of the pulmonary nodule revealed histopathology consistent with spindle cell sarcoma thought to originate in the right atrium. Immunohistochemical stains confirmed that this was an angiosarcoma. There was no evidence of extracardiac origin of the tumour. The patient was treated with chemotherapy and radiation. This case highlights the clinical presentation, rapid
\end{abstract}

and aggressive course of cardiac angiosarcomas, and the diagnostic modalities available for accurate diagnosis.

(Heart 1999;81:556-558)

Keywords: angiosarcoma; tumours; spindle cell carcinoma

Cardiac tumours are extremely uncommon and are mostly secondaries from other viscera. Sarcomas are the most common primary malignant cardiac tumours of which angiosarcoma is the most common and most aggressive histological entity. Unless there is a high clinical suspicion for these entities most are missed and detected at very late stages. A good clinical examination, appropriate use of diagnostic modalities, aggressive surgical treatment along with chemotherapy and radiation may improve survival in a patient with otherwise dismal prognosis. Novel approaches such as the use of interleukin- 2 have been reported and cardiac transplantation has been done in unresectable cases. Primary cardiac malignancies are rare and most are diagnosed by imaging or at necropsy. This case of a 29 year old white man highlights the clinical presentation, rapid and aggressive course of cardiac angiosarcomas, and the diagnostic modalities available for accurate diagnosis.
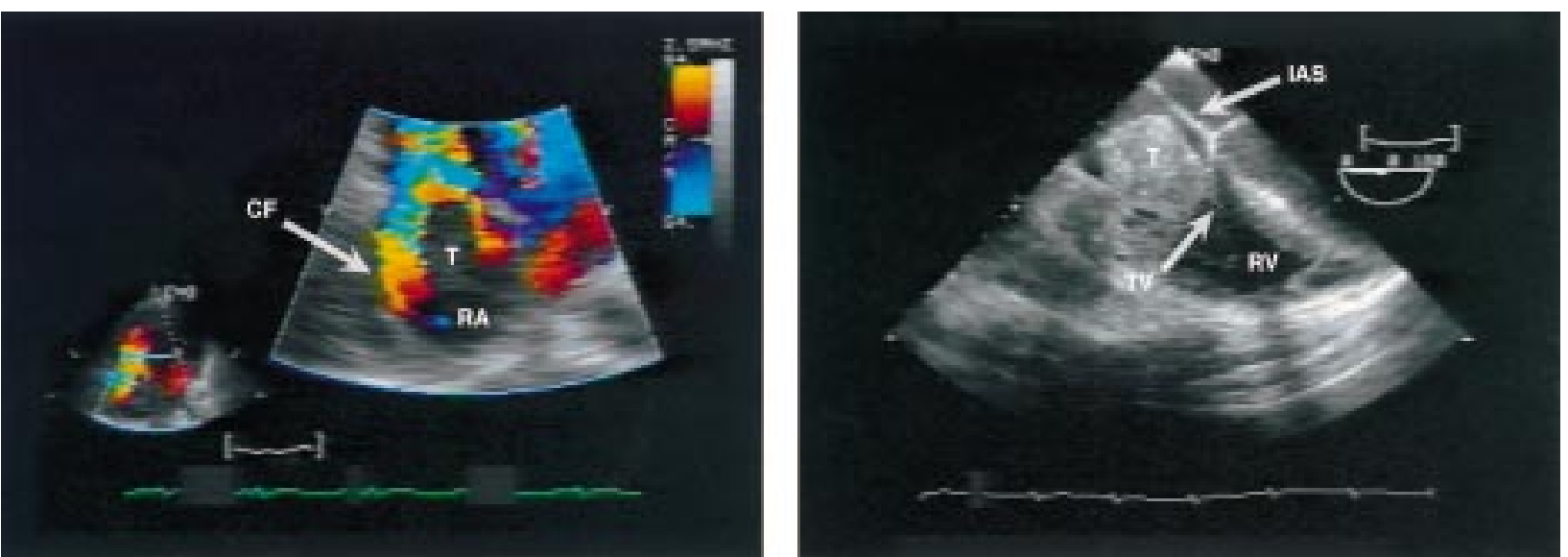

Figure 1 (Left) Four chamber view transthoracic echocardiogram focusing on colour flow (CF) across the tricuspid valve. The turbulent flow encircles the tumour (T) in the right atrium (RA) and was responsible for the mid-diastolic murmur of tricuspid stenosis. (Right) Transoesophageal echocardiogram showing the tumour (T) prolapsing across the tricuspid valve (TV) in diastole. IAS, interventricular septum; RV, right ventricle. 

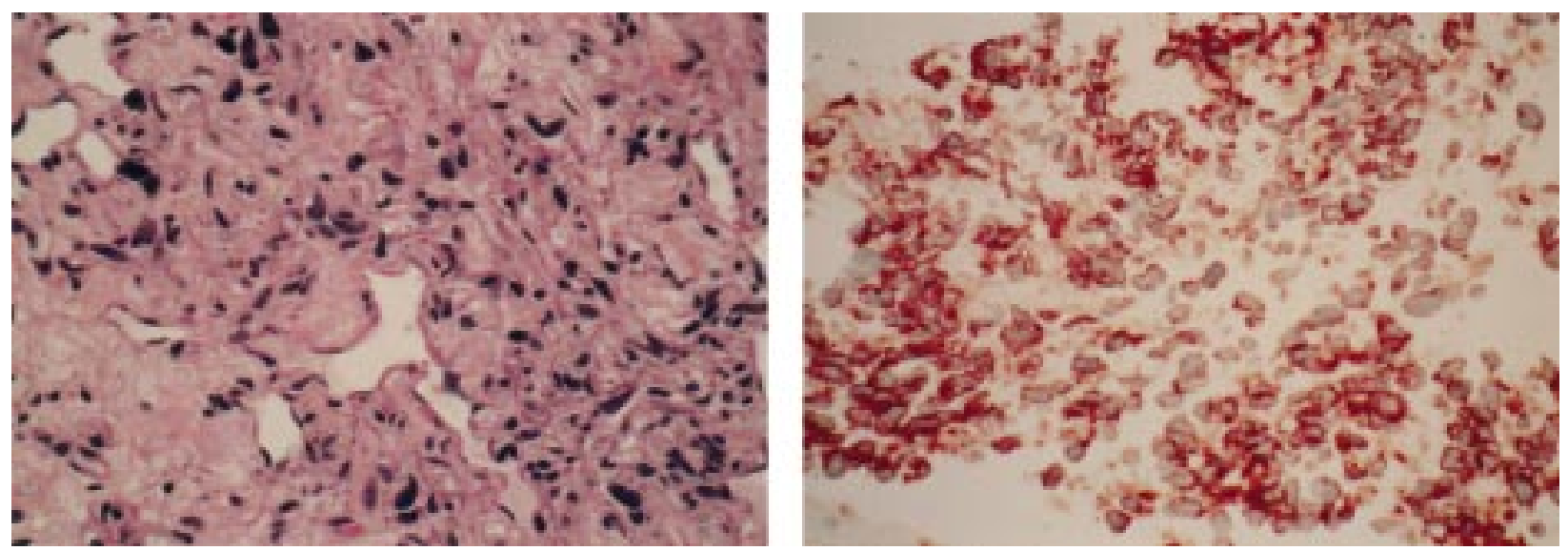

Figure 2 (Left) Haematoxylin and eosin stain of lung metastasis showing pleomorphic spindle cells with irregular nuclei lining the vascular channels containing red blood cells highly suggestive of angiosarcoma. (Right) Immunoperoxidase stain showing malignant cells strongly positive for CD31, indicative of the vascular origin of the tumour.

\section{Case report}

A 29 year old white man presented to the emergency room with new onset pleuritic chest pain and shortness of breath. His physical examination revealed a faint pericardial rub. Electrocardiography did not demonstrate diffuse ST abnormalities and laboratory data were within normal limits. Initial diagnostic work up included chest radiography, which revealed mild prominence of the right heart borders. Echocardiography showed normal left ventricular (LV) function and a small pericardial effusion. He was diagnosed as having viral pericarditis and was treated with non-steroidal anti-inflammatory drugs to which he responded well initially.

A few weeks later he developed recurrent chest pain with cough and haemoptysis. Chest radiography revealed prominence of right heart borders and vague multiple opacities in both lung fields. A ventilation-perfusion scan was negative for pulmonary embolism. Cardiac examination revealed a $2 / 6$ mid-diastolic murmur along the left sternal edge suggestive of tricuspid stenosis. Transthoracic echocardiography showed normal LV function, dilated right atrium and right ventricle. There was a $4-5 \mathrm{~cm}$ echodense mass in the right atrium adjacent to the interatrial septum and septal leaflet of the tricuspid valve (fig 1). Transoesophageal echocardiography further delineated that the mass $(8 \times 10 \mathrm{~cm})$ arose from the posterior wall of the right atrium, not attached to the interatrial septum, and protruded into the lumen of the right atrium causing intermittent obstruction of inflow across the tricuspid valve. To delineate the mass further, contrast computed tomography of the chest was done, which revealed a right atrial mass (diameter $7.8 \mathrm{~cm}$, width $8.4 \mathrm{~cm}$ ) extending to the anterior chest wall. The lung fields were studded with numerous pulmonary nodules suggestive of metastases.

A fine needle aspiration of the pulmonary nodule revealed histopathology consistent with spindle cell sarcoma thought to originate in the right atrium. Immunohistochemical stains confirmed that this was an angiosarcoma (fig 2). There was no evidence of extracardiac origin of the tumour. Due to the extent of the disease the patient was treated with chemotherapy and radiation during which he developed hemiparesis; computed tomography of the brain showed a solitary lesion in the left hemisphere consistent with metastasis. His hemiparesis resolved during chemotherapy and he continues to undergo treatment.

\section{Discussion}

Primary malignant cardiac tumours are very rare $^{1}$ and most cardiac neoplasia are secondaries from other viscera. A quarter of cardiac tumours exhibit histological evidence of malignancy. Sarcomas are the most common cardiac neoplasm of which angiosarcoma is the most common malignant variety. They form $75 \%$ of malignant tumours, the rest being mesotheliomas, lymphomas, and other less common neoplasia. Common age of presentation is the third to fifth decade. Unexplained chest pain, haemoptysis, systolic or diastolic murmurs, clubbing, anaemia, cardiomegaly or metastatic manifestations may be the first clue to these rare yet devastating entities. The right atrium is the most common site of origin followed by the left atrium, right ventricle and left ventricle, and the interventricular septum. Clinical manifestations vary depending upon the size and location of the tumours rather than the histology per se. Atrial location presents with mitral or tricuspid inflow obstruction (as in our patient), superior or inferior vena cava obstruction, systemic or pulmonary emboli, supraventricular arrhythmias or tamponade. Ventricular involvement of neoplasia presents with heart failure, ventricular arrhythmias, embolic events or tamponade. Patients with cardiac neoplasia may have conduction abnormalities, malignant ventricular arrhythmias from myocardial infiltration, and low voltage complexes from pericardial infiltration. Close correlation has been demonstrated between ECG lead abnormalities and the anatomic location of the tumour.

Most angiosarcomas are metastasised at the time of diagnosis and have a poor prognosis of 2-24 months from the time of detection. ${ }^{23}$ Sarcomas are derived from the mesenchyme and have predilection for men. Lesions of Kaposi's sarcoma primarily involving the heart 
have been described in anecdotal case reports. These usually involve the pericardium and are small and asymptomatic. Lack of attachment to the interatrial septum and mass originating from the posterior wall of the atrium should raise suspicion of a neoplastic process, apart from the rapidity of progression. Although transthoracic echocardiography can help in the diagnosis of these entities, transoesophageal echocardiography is clearly superior to delineate dimensions, origin, and extension of the tumours. ${ }^{4}$ Ultrafast computed tomography ${ }^{5}$ and magnetic resonance imaging (MRI $)^{6}$ have excellent diagnostic advantages over echocardiography with regards to tumour delineation and spread but are yet to be evaluated in controlled trials. Spin-echo MRI helps to delineate the extent of the mass in the cardiac chambers, as well as its attachments and effects on myocardial and valvar function. Surgery is the main stay of treatment for sarcomas and other primary cardiac malignancies, although success is limited by early recurrence and spread. ${ }^{7}$ If detected early and the tumour is localised, debulking surgery may be attempted. In one series, postoperative survival ranged from 2-55 months (mean 14). ${ }^{8}$

Owing to the extreme malignant potential and widespread nature of angiosarcomas at the time of diagnosis, treatment options are sometimes limited to palliative therapy and radiation to which they have variable response. Postoperative mediastinal radiation with average doses in the range of 5520 rads have been said to prolong survival. ${ }^{8}$ There are anecdotal reports of response of angiosarcoma to recom- binant interleukin- $2^{9}$ although not curative. Interleukin-2 acts by activating natural killer cells and cytotoxic $\mathrm{T}$ lymphocytes, which may help in arresting growth of the tumour. In rare instances of unresectable tumours, cardiac transplantation has been done ${ }^{1011}$ although recurrence of the tumour resulting from immunosuppression has thwarted success. ${ }^{12}$

We thank Dr Anil Prasad (Department of Pathology, Henry
Ford Hospital) for his contribution and guidance in the prepaFord Hospital) for his contribution and guidance
ration and interpretation of the pathology slides.

1 Allard MF, Taylor GP, Wilson JE, et al. Primary cardiac tumors. In: Goldhaber SZ, Braunwald E, eds. Cardiopulmonary diseases and cardiac tumors. atlas of heart disease, vol 3 . Phildelphia: Current Medicine, 1995:15.1-15.22.

2 Whorton CM. Primary malignant tumors of the heart. Cancer 1949;2:245.

3 Sorlie DAG, Myhre ESP, Stalsberg H. Angiosarcoma of the heart: unusual presentation and survival after treatment. $\mathrm{Br}$ heart: unusual presentatio

4 Edwards LC III, Louise EK. Transthoracic and transesophageal echocardiography for the evaluation of cardiac tumors, thrombi, and valvular regurgitation. Am $f$ Card Imag 1994;8:45.

5 Lipton MJ. Quantitation of cardiac function by cine-CT. Radiol Clin North Am 1985;23:613.

6 Lund JT, Ehman RL, Julsrud PR, et al. Cardiac masses: assessment by MR imaging. Am F Roetgenol 1989;152:46973 .

7 Bleisch N, Kraus F. Polypoid sarcoma of the pulmonary trunk. Cancer 1980;6:314.

8 Poole GV Jr, Meredith JW, Breyer RH, et al. Surgical implications in malignant cardiac diseases. Ann Thorac Surg 1983;36:484-91.

9 Kakizaki S, Takagi H, Hosaka Y. Cardiac angiosarcoma responding to multidisciplinary treatment. Int $\mathcal{f}$ Cardiol responding to mul.

10 Baay P, Karwande SV, Kushner JP, et al. Succesful treatment of cardiac angiosarcoma with combined modality therapy. $\mathcal{F}$ Heart Lung Transplant 1994;13:923-5.

11 Matheis G, Beyersdorf F. Primary cardiac angiosarcoma. A case report. Cardiology 1995;86:83-5.

12 Seinbenmann R, Jenni R, Makek M. Primary synovial sarcoma of the heart treated by cardiac transplantation. $\mathcal{f}$ Thorac Cardiovasc Surg 1990;99:567-8. 